\title{
Advances in radiation therapy for thoracic malignancies
}

This special issue of fournal of Thoracic Disease (FTD) is a focused review of advances in radiation oncology for thoracic malignancies. The issue covers advances in technology relevant to the practice of radiation oncology in the modern era, as well as the most current treatment approaches for early-stage, advanced, recurrent and oligometastatic non-small cell lung cancer (NSCLC), while recognizing the importance of tumor biology, radiation fractionation, immunotherapy, and functional imaging in the modern treatment of NSCLC. The issue ends with reviews of current data and treatment approaches for small cell lung cancer (SCLC), thymic malignancies, and malignant pleural mesothelioma.

More specifically, the first article of the focused issue pertains to image guidance and motion management and mitigation. Molitoris and colleagues detail how the advent of and improvements in image-guided radiation therapy (IGRT) have enabled the increased utilization of advanced radiation modalities like intensity-modulated radiation therapy (IMRT), stereotactic body radiation therapy (SBRT), and proton therapy for the treatment of lung cancer. IGRT is the use of imaging during or just prior to radiotherapy delivery that verifies the agreement of anatomy between the treatment plan and the patient's actual setup on the treatment table (1). While improving accuracy of treatment delivery by allowing visualization of the tumor on the treatment table, IGRT can also help inform for the need of adaptive replanning if notable anatomical changes (tumor or normal tissue) from the time of planning simulation are identified on daily imaging. Furthermore, thoracic tumors are unique in their susceptibility to motion with respiration, leading to uncertainties in target and normal tissue positioning. Motion management strategies, including motion encompassment, respiratory gating, and breath-hold methods, as well as motion mitigation strategies, including respiratory coaching, abdominal compression, and tumor tracking, are explained. Collectively, the authors detail how target volume expansions for both motion and set-up uncertainties can be minimized with motion management/ mitigation strategies and with IGRT, allowing for better sparing of irradiation dose to adjacent critical structures.

Sebastian and colleagues next discuss contemporary insights and the latest advances in SBRT for early stage NSCLC. SBRT has emerged as the standard of care for patients with medically inoperable early stage NSCLC (2). Given its favorable toxicity profile and high rates of local control (3), which have allowed for population-based improvements in overall survival for NSCLC (4), interest in SBRT for operable patients has increased, with early results of under-accrued clinical trials suggesting at least equipoise for SBRT compared with surgery $(5,6)$. The authors discuss practical questions and active areas of investigation for the still relatively young thoracic modality, including delivery technique, total radiation dose, dosefractionation regimen, and timing of treatments, and they describe how these can be impacted based on tumor size, location, histology, and molecular phenotype.

Next, Roach and colleagues from Washington University School of Medicine review the optimal radiation dose and fractionation for treating locally advanced NSCLC. While the standard of care for medically inoperable or surgically unresectable patients is concurrent chemoradiation (7), local failure rates are significant after chemoradiation and are a major driver of death from lung cancer (8). One approach to counter these high local failure rates has been to escalate the total radiation dose. While this strategy showed promise in smaller, earlier phase studies $(9,10)$, the recently reported RTOG 0617 phase III randomized demonstrated a survival decrement to unselective dose escalation to 74 Gy, largely due to increased toxicities with this higher dose (11). The authors describe other dosing strategies that have been investigated to overcome the high local failure rates of NSCLC and to minimize tumor repopulation during treatment, including accelerated hyperfractionation, hypofractionation, and the use of metabolic imaging during treatment to boost areas of residual disease.

Yegya-Raman et al. discuss advanced radiation techniques for locally advanced NSCLC, including IMRT and proton therapy. By potentially reducing normal tissue toxicities, these modalities may allow for safer concurrent chemoradiation dose escalation for unresectable patients. This may also be important in operable patients, where dose escalation of neoadjuvant irradiation may be associated with improved outcomes for trimodality NSCLC patients (12). With IMRT, the fluence of radiation across each beam is modified, thus allowing more conformal treatment delivery and better sparing of organs at risk near the target volume. Compared with 3CRT, retrospective studies have shown that IMRT can reduce the rate of radiation pneumonitis and even improve overall survival $(13,14)$. Although a survival benefit was not seen with IMRT on secondary analysis of RTOG 0617, IMRT compared with 3DCRT as a preplanned stratification factor did allow for better preservation of quality of life, reduced rates of radiation pneumonitis, reduced cardiac irradiation doses, and better compliance with 
chemotherapy $(15,16)$. With proton therapy, radiation can be deposited at a specific depth, after which the protons decelerate rapidly, allowing for dose deposition to the tumor and little to no dose beyond the tumor (17). Compared with photon therapy, including IMRT, proton therapy allows for better sparing of organs at risk $(18,19)$. While single-arm prospective studies have reported lower rates of pneumonitis and esophagitis with proton therapy than would be expected with photon therapy $(20,21)$, and national registry data have demonstrated a survival benefit with proton therapy (22), a recently reported randomized trial failed to show a significant benefit of proton therapy over IMRT in terms of pneumonitis or local control (23). The authors discuss indications for both IMRT and proton therapy, which patients might benefit most, implementation challenges, and key trials currently enrolling accessing these advanced modalities.

Badiyan and colleagues next report on a hot topic in thoracic oncology_combining radiation therapy with immunotherapy. While immunotherapy has clearly established itself as a standard treatment modality for advanced NSCLC $(24,25)$, its role in early stage and locally advanced NSCLC is still being defined, with a highly promising early report from the PACIFIC trial demonstrating that maintenance durvalumab given after chemoradiation for locally advanced NSCLC tripled the progression free survival and improve the time to death or distant metastasis (26). As radiation therapy can mount a robust anti-tumor immune response, it has been hypothesized to have the potential to work synergistically with immunotherapy $(27,28)$. In fact, preclinical data support synergy between radiation therapy and immunotherapy (29). This manuscript reviews the preclinical rationale for combining radiotherapy with immunotherapy, the clinical data to date on the combination of radiotherapy and immunotherapy across thoracic malignancies, and the ongoing clinical trials investigating the combination of radiation therapy and immunotherapy for thoracic cancers.

Positron emission tomography/computed tomography (PET/CT) has established itself as an essential part of diagnosis and staging for NSCLC, as a useful modality for monitoring treatment response following radiotherapy (30). Konert et al. detail how PET has an increasingly important role in prognostication and in radiation target volume delineation (31). The authors detail how PET metrics like standardized uptake value, total lesion glycolysis, and other functional measures have been shown to be prognostic for NSCLC (32). They also discuss how PET radiomics textural features can improve prognostication. Additional PET tracers and other future areas of research are also detailed. Furthermore, as advanced radiation modalities have allowed for more precise treatment delivery, accurate tumor delineation for thoracic malignancies is more critical than ever. The authors next discuss how PET can aid in target volume delineation, and they describe automatic target delineation using automated segmentation and other techniques.

Vyfhuis and colleagues report on reirradiation for locoregionally recurrent NSCLC. As previously discussed, locoregional recurrences in patients with locally advanced NSCLC are quite common. Standard treatment approaches for such recurrences have generally focused on non-curative systemic options like cytotoxic chemotherapy or immunotherapy due to the concerns of potentially life-threatening complications than can be seen with thoracic reirradiation. All of the advances detailed above, including IGRT, SBRT, IMRT and proton therapy, have allowed for potentially safer reirradiation options for recurrent NSCLC. The authors discuss conventional photon and SBRT reirradiation, as well as proton reirradiation, a particularly attractive use of the modality due to its ability to minimize or eliminate dose to previously irradiated adjacent critical structures (33). In fact, two relatively large studies, one retrospective (34) and one prospective (35), have recently been reported showing the ability of proton therapy to be generally safely administered in the reirradiation setting, with encouraging survival outcomes.

Oligometastatic disease, often defined as limited metastatic disease to five or fewer sites, is increasingly being recognized as a distinct entity from more widespread metastatic disease with a unique prognosis that warrants a different treatment paradigm (36). This recognition has led to oligometastases being incorporated into the new AJCC $8^{\text {th }}$ edition staging manual. Investigators are now recognizing heterogeneity within oligometastatic patients, with patients having varied prognoses according to the number of sites with metastases, the specific sites of metastases, if metastases occur synchronously or metachronously, and if there are nodal metastases (37). Tumati and Iyengar describe how advances in imaging have allowed for a more apparent identification of a subset of patients with isolated metastatic deposits. They then discuss the implications of metastatic disease extent, detail the role of surgery for oligometastatic disease, and review the data for using radiotherapy and SBRT for oligometastases, including two recently reported randomized trials showing a benefit in progression free survival for local therapy compared with systemic therapy alone $(38,39)$. The also detail oligoprogressive disease and its management, and they discuss how the role of local therapy is evolving with the rise in immunotherapy for NSCLC. 
An international group of collaborators next discuss the evolving role of radiotherapy for SCLC. These authors discuss the long-standing question of once daily versus twice daily irradiation, the optimal dosing for once daily irradiation, the evidence for hypofractionation, the role of prophylactic cranial irradiation (PCI), and the impact PET/CT has had on staging for limited-stage SCLC. For extensive-stage SCLC they detail the data for (40) and against (41) PCI, and they summarize and put into context the recently reported CREST (42) and RTOG 0937 (43) trials of thoracic consolidative radiotherapy. They next discuss options for attempting to reduce neurocognitive dysfunction following PCI, including stereotactic radiosurgery and hippocampal avoidance whole brain radiation therapy. Finally, they discuss encouraging new data of advanced radiotherapy modalities like SBRT for stage I SCLC $(44,45)$ and proton therapy for locally advanced SCLC (46).

Willmann and Rimner next review the role of radiation therapy for thymic malignancies. The most established role of radiotherapy for this group of rare malignancies is in the adjuvant setting, especially for advanced or incompletely resected disease. However, as recent reports have demonstrated a survival benefit to adjuvant radiotherapy $(47,48)$, even for completely resection and early stage disease, a paradigm shift in the treatment approach for thymic malignancies is occurring and is described in this review. The roles of neoadjuvant radiotherapy for marginally resectable patients and definitive radiotherapy for unresectable patients are also detailed. Methods to reduce treatment toxicities for thymic malignancies, which generally have better prognoses than other thoracic malignancies, are critical, and data for IMRT (49) and proton therapy (50) are discussed.

The last article in this focused issue of $\mathcal{F T D}$ is on malignant pleural mesothelioma, another rare thoracic malignancy. Cramer and colleagues detail the unique challenges of delivering radiation therapy to large thoracic volumes that are often required for mesothelioma. Strategies of and data for employing radiation therapy before (51) or after (52) extrapleural pneumonectomy and after lung-sparing extended pleurectomy/decortication $(53,54)$ are discussed. The concept of definitive irradiation is also described (55). The authors then discuss the controversial role of adjuvant prophylactic radiotherapy to intervention sites used in an attempt to reduce the risk of surgical tract dissemination, as well as the more common role of palliative radiotherapy for patients with mesothelioma who often present with dyspnea and/or pain. Lastly, the authors discuss future potential advances in treatment for mesothelioma, including the use of proton therapy (56) and of combining radiation therapy with immunotherapy (57).

In reading the manuscripts in this focused issue, it is clear there have been tremendous advances in thoracic radiation oncology in the past decade. We fully anticipate the next decade will bring further practice-changing progress, and we look forward to the evolving data from active areas of investigation integrating the use of advanced radiation therapy technologies with biological response modifiers and immunotherapy, and with treatment guided by modern anatomical and functional imaging. These modern approaches will well position the next generation radiation oncologists to continue to improve outcomes for patients with thoracic malignancies.

\section{Acknowledgements}

None.

\section{References}

1. Cho BC, Bezjak A, Dawson LA. Image guidance in non-small cell lung cancer. Semin Radiat Oncol 2010;20:164-70.

2. Videtic GM, Donington J, Giuliani M, et al. Stereotactic body radiation therapy for early-stage non-small cell lung cancer: Executive Summary of an ASTRO Evidence-Based Guideline. Pract Radiat Oncol 2017;7:295-301.

3. Timmerman R, Paulus R, Galvin J, et al. Stereotactic body radiation therapy for inoperable early stage lung cancer. JAMA 2010;303:1070-6.

4. Boyer MJ, Williams CD, Harpole DH, et al. Improved Survival of Stage I Non-Small Cell Lung Cancer: A VA Central Cancer Registry Analysis. J Thorac Oncol 2017;12:1814-23.

5. Chang JY, Senan S, Paul MA, et al. Stereotactic ablative radiotherapy versus lobectomy for operable stage I non-small-cell lung cancer: a pooled analysis of two randomised trials. Lancet Oncol 2015;16:630-7.

6. Simone CB 2nd, Dorsey JF. Additional data in the debate on stage I non-small cell lung cancer: surgery versus stereotactic ablative radiotherapy. Ann Transl Med 2015;3:172. 
7. Curran WJ Jr, Paulus R, Langer CJ, et al. Sequential vs. concurrent chemoradiation for stage III non-small cell lung cancer: randomized phase III trial RTOG 9410. J Natl Cancer Inst 2011;103:1452-60.

8. Machtay M, Paulus R, Moughan J, et al. Defining local-regional control and its importance in locally advanced non-small cell lung carcinoma. J Thorac Oncol 2012;7:716-22.

9. Yuan S, Sun X, Li M, et al. A randomized study of involved-field irradiation versus elective nodal irradiation in combination with concurrent chemotherapy for inoperable stage III nonsmall cell lung cancer. Am J Clin Oncol 2007;30:239-44.

10. Bradley J, Graham MV, Winter K, et al. Toxicity and outcome results of RTOG 9311: a phase I-II dose-escalation study using three-dimensional conformal radiotherapy in patients with inoperable non-small-cell lung carcinoma. Int J Radiat Oncol Biol Phys 2005;61:318-28.

11. Bradley JD, Paulus R, Komaki R, et al. Standard-dose versus high-dose conformal radiotherapy with concurrent and consolidation carboplatin plus paclitaxel with or without cetuximab for patients with stage IIIA or IIIB non-small-cell lung cancer (RTOG 0617): a randomised, two-by-two factorial phase 3 study. Lancet Oncol 2015;16:187-99.

12. Vyfhuis MA, Bhooshan N, Burrows WM, et al. Oncological outcomes from trimodality therapy receiving definitive doses of neoadjuvant chemoradiation $(\geq 60 \mathrm{~Gy})$ and factors influencing consideration for surgery in stage III non-small cell lung cancer. Adv Radiat Oncol 2017;2:259-69.

13. Yom SS, Liao Z, Liu HH, et al. Initial evaluation of treatment-related pneumonitis in advanced-stage non-small-cell lung cancer patients treated with concurrent chemotherapy and intensity-modulated radiotherapy. Int J Radiat Oncol Biol Phys 2007;68:94-102.

14. Liao ZX, Komaki RR, Thames HD Jr, et al. Influence of technologic advances on outcomes in patients with unresectable, locally advanced non-small-cell lung cancer receiving concomitant chemoradiotherapy. Int J Radiat Oncol Biol Phys 2010;76:775-81.

15. Movsas B, Hu C, Sloan J, et al. Quality of Life Analysis of a Radiation Dose-Escalation Study of Patients With Non-Small-Cell Lung Cancer: A Secondary Analysis of the Radiation Therapy Oncology Group 0617 Randomized Clinical Trial. JAMA Oncol 2016;2:359-67.

16. Chun SG, Hu C, Choy H, et al. Impact of Intensity-Modulated Radiation Therapy Technique for Locally Advanced NonSmall-Cell Lung Cancer: A Secondary Analysis of the NRG Oncology RTOG 0617 Randomized Clinical Trial. J Clin Oncol 2017;35:56-62.

17. Chang JY, Jabbour SK, De Ruysscher D, et al. Consensus Statement on Proton Therapy in Early-Stage and Locally Advanced Non-Small Cell Lung Cancer. Int J Radiat Oncol Biol Phys 2016;95:505-16.

18. Giaddui T, Chen W, Yu J, et al. Establishing the feasibility of the dosimetric compliance criteria of RTOG 1308: phase III randomized trial comparing overall survival after photon versus proton radiochemotherapy for inoperable stage II-IIIB NSCLC. Radiat Oncol 2016;11:66.

19. Kesarwala AH, Ko CJ, Ning H, et al. Intensity-modulated proton therapy for elective nodal irradiation and involved-field radiation in the definitive treatment of locally advanced non-small-cell lung cancer: a dosimetric study. Clin Lung Cancer 2015;16:237-44.

20. Chang JY, Komaki R, Lu C, et al. Phase 2 study of high-dose proton therapy with concurrent chemotherapy for unresectable stage III nonsmall cell lung cancer. Cancer 2011;117:4707-13.

21. Chang JY, Verma V, Li M, et al. Proton Beam Radiotherapy and Concurrent Chemotherapy for Unresectable Stage III NonSmall Cell Lung Cancer: Final Results of a Phase 2 Study. JAMA Oncol 2017;3:e172032.

22. Higgins KA, O'Connell K, Liu Y, et al. National Cancer Database Analysis of Proton Versus Photon Radiation Therapy in Non-Small Cell Lung Cancer. Int J Radiat Oncol Biol Phys 2017;97:128-37.

23. Liao Z, Lee JJ, Komaki R, et al. Bayesian Adaptive Randomization Trial of Passive Scattering Proton Therapy and IntensityModulated Photon Radiotherapy for Locally Advanced Non-Small-Cell Lung Cancer. J Clin Oncol 2018;36:1813-22.

24. Brahmer J, Reckamp KL, Baas P, et al. Nivolumab versus Docetaxel in Advanced Squamous-Cell Non-Small-Cell Lung Cancer. N Engl J Med 2015;373:123-35.

25. Borghaei H, Paz-Ares L, Horn L, et al. Nivolumab versus Docetaxel in Advanced Nonsquamous Non-Small-Cell Lung Cancer. N Engl J Med 2015;373:1627-39.

26. Antonia SJ, Villegas A, Daniel D, et al.; PACIFIC Investigators. Durvalumab after Chemoradiotherapy in Stage III Non-SmallCell Lung Cancer. N Engl J Med 2017;377:1919-29.

27. Simone CB 2nd, Burri SH, Heinzerling JH. Novel radiotherapy approaches for lung cancer: combining radiation therapy with 
targeted and immunotherapies. Transl Lung Cancer Res 2015;4:545-52.

28. Simone CB 2nd, Berman AT, Jabbour SK. Harnessing the potential synergy of combining radiation therapy and immunotherapy for thoracic malignancies. Transl Lung Cancer Res 2017;6:109-12.

29. Deng L, Liang H, Burnette B, et al. Irradiation and anti-PD-L1 treatment synergistically promote antitumor immunity in mice. J Clin Invest 2014;124:687-95.

30. Simone CB 2nd, Houshmand S, Kalbasi A, et al. PET-Based Thoracic Radiation Oncology. PET Clin 2016;11:319-32.

31. Verma V, Choi JI, Sawant A, et al. Use of PET and Other Functional Imaging to Guide Target Delineation in Radiation Oncology. Semin Radiat Oncol 2018;28:171-7.

32. Salavati A, Duan F, Snyder BS, et al. Optimal FDG PET/CT volumetric parameters for risk stratification in patients with locally advanced non-small cell lung cancer: results from the ACRIN 6668/RTOG 0235 trial. Eur J Nucl Med Mol Imaging 2017;44:1969-83.

33. Verma V, Rwigema JM, Malyapa RS, et al. Systematic assessment of clinical outcomes and toxicities of proton radiotherapy for reirradiation. Radiother Oncol 2017;125:21-30.

34. McAvoy S, Ciura K, Wei C, et al. Definitive reirradiation for locoregionally recurrent non-small cell lung cancer with proton beam therapy or intensity modulated radiation therapy: predictors of high-grade toxicity and survival outcomes. Int J Radiat Oncol Biol Phys 2014;90:819-27.

35. Chao HH, Berman AT, Simone CB 2nd, et al. Multi-Institutional Prospective Study of Reirradiation with Proton Beam Radiotherapy for Locoregionally Recurrent Non-Small Cell Lung Cancer. J Thorac Oncol 2017;12:281-92.

36. Ashworth A, Rodrigues G, Boldt G, et al. Is there an oligometastatic state in non-small cell lung cancer? A systematic review of the literature. Lung Cancer 2013;82:197-203.

37. Ashworth AB, Senan S, Palma DA, et al. An individual patient data metaanalysis of outcomes and prognostic factors after treatment of oligometastatic non-small-cell lung cancer. Clin Lung Cancer 2014;15:346-55.

38. Gomez DR, Blumenschein GR Jr, Lee JJ, et al. Local consolidative therapy versus maintenance therapy or observation for patients with oligometastatic non-small-cell lung cancer without progression after first-line systemic therapy: a multicentre, randomised, controlled, phase 2 study. Lancet Oncol 2016;17:1672-82.

39. Iyengar P, Wardak Z, Gerber DE, et al. Consolidative Radiotherapy for Limited Metastatic Non-Small-Cell Lung Cancer: A Phase 2 Randomized Clinical Trial. JAMA Oncol 2018;4:e173501.

40. Slotman B, Faivre-Finn C, Kramer G, et al. Prophylactic cranial irradiation in extensive small-cell lung cancer. N Engl J Med 2007;357:664-72.

41. Takahashi T, Yamanaka T, Seto T, et al. Prophylactic cranial irradiation versus observation in patients with extensive-disease small-cell lung cancer: a multicentre, randomised, open-label, phase 3 trial. Lancet Oncol 2017;18:663-71.

42. Slotman BJ, van Tinteren H, Praag JO, et al. Use of thoracic radiotherapy for extensive stage small-cell lung cancer: a phase 3 randomised controlled trial. Lancet 2015;385:36-42.

43. Gore EM, Hu C, Sun AY, et al. Randomized Phase II Study Comparing Prophylactic Cranial Irradiation Alone to Prophylactic Cranial Irradiation and Consolidative Extracranial Irradiation for Extensive-Disease Small Cell Lung Cancer (ED SCLC): NRG Oncology RTOG 0937. J Thorac Oncol 2017;12:1561-70.

44. Verma V, Simone CB 2nd, Allen PK, et al. Multi-Institutional Experience of Stereotactic Ablative Radiation Therapy for Stage I Small Cell Lung Cancer. Int J Radiat Oncol Biol Phys 2017;97:362-71.

45. Verma V, Simone CB 2nd, Allen PK, et al. Outcomes of Stereotactic Body Radiotherapy for T1-T2N0 Small Cell Carcinoma According to Addition of Chemotherapy and Prophylactic Cranial Irradiation: A Multicenter Analysis. Clin Lung Cancer 2017;18:675-81.e1.

46. Rwigema JM, Verma V, Lin L, et al. Prospective study of proton-beam radiation therapy for limited-stage small cell lung cancer. Cancer 2017;123:4244-51.

47. Rimner A, Yao X, Huang J, et al. Postoperative Radiation Therapy Is Associated with Longer Overall Survival in Completely Resected Stage II and III Thymoma-An Analysis of the International Thymic Malignancies Interest Group Retrospective Database. J Thorac Oncol 2016;11:1785-92.

48. Jackson MW, Palma DA, Camidge DR, et al. The Impact of Postoperative Radiotherapy for Thymoma and Thymic Carcinoma. J Thorac Oncol 2017;12:734-44.

49. Fan C, Feng Q, Chen Y, et al. Postoperative radiotherapy for completely resected Masaoka stage III thymoma: a retrospective 
study of 65 cases from a single institution. Radiat Oncol 2013;8:199.

50. Vogel J, Berman AT, Lin L, et al. Prospective study of proton beam radiation therapy for adjuvant and definitive treatment of thymoma and thymic carcinoma: Early response and toxicity assessment. Radiother Oncol 2016;118:504-9.

51. de Perrot M, Feld R, Leighl NB, et al. Accelerated hemithoracic radiation followed by extrapleural pneumonectomy for malignant pleural mesothelioma. J Thorac Cardiovasc Surg 2016;151:468-73.

52. Gomez DR, Hong DS, Allen PK, et al. Patterns of failure, toxicity, and survival after extrapleural pneumonectomy and hemithoracic intensity-modulated radiation therapy for malignant pleural mesothelioma. J Thorac Oncol 2013;8:238-45.

53. Rimner A, Zauderer MG, Gomez DR, et al. Phase II Study of Hemithoracic Intensity-Modulated Pleural Radiation Therapy (IMPRINT) As Part of Lung-Sparing Multimodality Therapy in Patients With Malignant Pleural Mesothelioma. J Clin Oncol 2016;34:2761-8.

54. Friedberg JS, Simone CB 2nd, Culligan MJ, et al. Extended Pleurectomy-Decortication-Based Treatment for Advanced Stage Epithelial Mesothelioma Yielding a Median Survival of Nearly Three Years. Ann Thorac Surg 2017;103:912-9.

55. Feigen M, Lee ST, Lawford C, et al. Establishing locoregional control of malignant pleural mesothelioma using high-dose radiotherapy and (18) F-FDG PET/CT scan correlation. J Med Imaging Radiat Oncol 2011;55:320-32.

56. Badiyan SN, Molitoris JK, Zhu M, et al. Proton beam therapy for malignant pleural mesothelioma. Transl Lung Cancer Res 2018;7:189-98.

57. Alley EW, Katz SI, Cengel KA, et al. Immunotherapy and radiation therapy for malignant pleural mesothelioma. Transl Lung Cancer Res 2017;6:212-9.

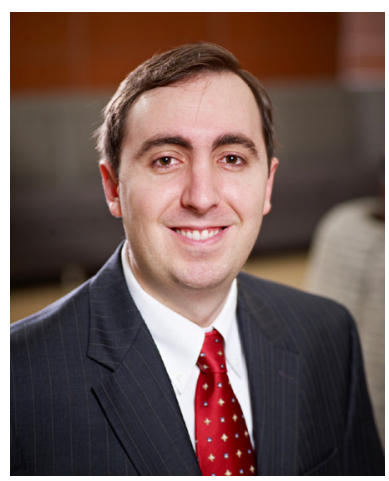

Charles B. Simone II

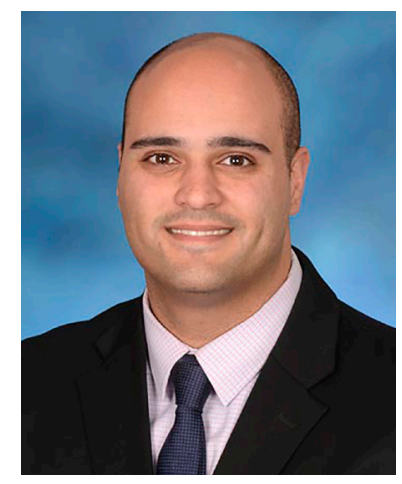

Shahed N. Badiyan

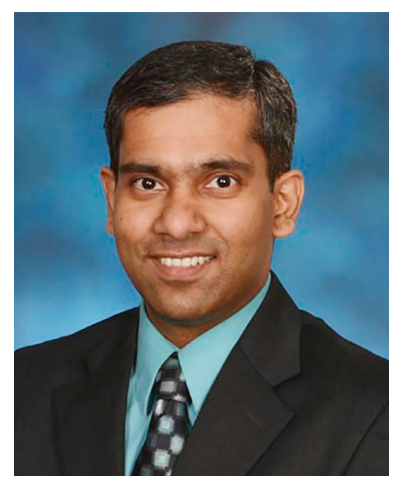

Pranshu Mohindra

Charles B. Simone II
Department of Radiation Oncology, University of Maryland School of Medicine, Baltimore, Maryland, USA.
(Email: charlessimone@umm.edu)
Shahed N. Badiyan
Department of Radiation Oncology, Washington University School of Medicine, St. Louis, Missouri, USA.
(Email: shabedbadiyan@gmail.com)

Pranshu Mohindra

Department of Radiation Oncology, University of Maryland School of Medicine, Baltimore, Maryland, USA.

(Email:pmobindra@umm.edu)

doi: $10.21037 /$ jtd.2018.08.10

Conflicts of Interest: The authors have no conflicts of interest to declare.

Cite this article as: Simone CB 2nd, Badiyan SN, Mohindra P. Advances in radiation therapy for thoracic malignancies. J Thorac Dis 2018;10(Suppl 21):S2431-S2436. doi: 10.21037/jtd.2018.08.10 